\title{
ALGEBRAIC STRUCTURE IN COMPLEX FUNCTION SPACES
}

\author{
A. J. ELLIS
}

(Communicated by William J. Davis)

\begin{abstract}
Let $M$ be a complex function space containing constants, and let $Z$ be the complex state space of $M$. If $M$ is linearly isometric to a uniform algebra and if $Z$ is affinely homeomorphic to the complex state space of a uniform algebra then we prove that $M$ is a uniform algebra. Neither of the two conditions taken separately imply this conclusion.
\end{abstract}

If $X$ is a compact Hausdorff space then $C_{\mathbf{C}}(X), C_{\mathbf{R}}(X)$ will denote the Banach spaces of all continuous complex-valued, respectively real-valued, functions on $X$ with the supremum norm. A closed linear subspace $M$ of $C_{\mathbf{C}}(X)$ which contains constants and separates the points of $X$ will be called a complex function space. The subset $S=\left\{\varphi \in M^{*}:\|\varphi\|=1=\varphi(1)\right\}$ of $M^{*}$ is called the state space of $M$ and the subset $Z=\operatorname{co}(S \cup-i S)$ of $M^{*}$ is called the complex state space of $M$; the sets $S$ and $Z$ are compact convex sets when endowed with the relative $w^{*}$-topology. If $K$ is any compact convex subset of a locally convex Hausdorff space then $A(K), A_{\mathrm{C}}(K)$ will denote the Banach spaces of all continuous real-valued, respectively complex-valued, affine functions on $K$ with the supremum norm.

We shall be concerned with the linear and norm structure of $M$ and the affine and topological structure of $Z$ and will seek conditions which imply that $M$ is a uniform algebra on $X$. To this end we say that the complex state spaces $Z_{1}, Z_{2}$ of two complex function spaces $M_{1}, M_{2}$ are equivalent if they are affinely homeomorphic, and that $Z_{1}, Z_{2}$ are real-equivalent if there is an affine homeomorphism $\eta: Z_{1} \rightarrow Z_{2}$ which maps $S_{1}$ onto $S_{2}$ (and hence maps $-i S_{1}$ onto $\left.-i S_{2}\right)$.

We begin by developing [4, Examples 3 and 1] to show that the property that $M$ has the linear and norm structure of a uniform algebra is independent from the property that $Z$ is equivalent (or real-equivalent) to the complex state space of a uniform algebra.

Received by the editors May 21, 1988.

1980 Mathematics Subject Classification (1985 Revision). Primary 46E15, 46J10.

Key words and phrases. Complex state space; linear isometry. 
Example 1. Let $M_{1}=P(\Gamma)$ be the disc algebra on the unit circle $\Gamma$, and let $M=\left\{\bar{z} f(z): f \in M_{1}\right\}$. Then [4, Example 3] shows that $M$ and $M_{1}$ are isometrically isomorphic while $Z$ and $Z_{1}$ are not equivalent. We will show that $Z$ is not equivalent to $Z_{2}$ for any uniform algebra $M_{2}$.

Suppose that $Z$ is equivalent to $Z_{2}$. Then the connectedness of $\Gamma$ implies that either $Z$ is real-equivalent to $Z_{2}$ or $Z$ is real-equivalent to the complex state space of $\bar{M}_{2}$ (cf. [5]). We may hence assume that $Z$ and $Z_{2}$ are realequivalent, and that $M_{2}$ is a uniform algebra on $\Gamma$ (cf. [4]). We have $M_{2}=$ $\{u+i v \circ \psi: u+i v \in M\}$, where $\psi: \Gamma \rightarrow \Gamma$ is a homeomorphism.

Now $z$ and $\bar{z}$ belong to $M$ and so the functions $f(z)=x+\operatorname{im} \psi^{\prime}(z)$ and $\bar{f}(z)=x-\operatorname{im} \psi(z)$ belong to $M_{2}$, where we write $z=x+i y$. Hence the function $g(z)=x$ belongs to $M_{2}$ and similarly, using the facts that $i z$ and $i \bar{z}$ belong to $M$, we see that the function $h(z)=y$ belongs to $M_{2}$. Consequently the uniform algebra $M_{2}$ equals $C_{\mathbf{C}}(\Gamma)$. This implies that $M=C_{\mathbf{C}}(\Gamma)$, giving the required contradiction.

Example 2. Let $M_{1}=P(\Gamma)$ and $M=\{f: f(z)=u(z)+i v(-z)$ for some $\left.u+i v \in M_{1}\right\}$. Then [4, Example 1] shows that $Z$ and $Z_{1}$ are real-equivalent while $M$ and $M_{1}$ are not isometrically isomorphic. We will show that $M$ is not isometrically isomorphic to any uniform algebra $M_{2}$.

If $M$ is isometrically isomorphic to $M_{2}$, a uniform algebra on $X$, then we will have $M_{2}=\{\lambda(f \circ \tau): f \in M\}$, where $\lambda \in M_{2}$ with $|\lambda|=1$ and $\tau: X \rightarrow \Gamma$ a homeomorphism. Writing $M_{3}=\left\{g \circ \tau^{-1}: g \in M_{2}\right\}$ we note that $M_{3}$ is a uniform algebra on $\Gamma$ equal to $\left\{\left(\lambda \circ \tau^{-1}\right) f: f \in M\right\}=\{l f: f \in M\}$, where $l=\lambda \circ \tau^{-1} \in M_{3}$ with $|l|=1$. Since $z^{2 n}$ and $\bar{z}^{2 n+1}$ belong to $M$, $n \geq 0$, the functions $l(z) z^{2 n}$ and $l(z) z^{2 n+1}$ belong to $M_{3}$. Since $M_{3}$ is an algebra and since $l(z)^{2} z^{2 n}=l(z)\left(l(z) z^{2 n}\right), l(z)^{2} \bar{z}^{2 n+1}=l(z)\left(l(z) \bar{z}^{2 n+1}\right)$, $l(z)^{2} z^{2 n+1}=(l(z) \bar{z})\left(l(z) z^{2 n+2}\right), l(z)^{2} \bar{z}^{2 n}=(l(z) \bar{z})\left(l(z) \bar{z}^{2 n+1}\right)$ we see that the functions $l(z)^{2} z^{k}, k$ any integer, belong to $M_{3}$. Since the polynomials in $z$ and $\bar{z}$ form a dense linear subspace of $C_{\mathbf{C}}(\Gamma)$ it follows that $M_{3}=C_{\mathbf{C}}(\Gamma)$. This implies that $M=C_{\mathrm{C}}(\Gamma)$, giving the required contradiction.

We will now show that if $M$ has the linear and norm structure of a uniform algebra, and if $Z$ is equivalent to the complex state space of a uniform algebra, then $M$ is necessarily a uniform algebra. We note firstly however that we cannot replace 'complex state space' by 'state space' in this result. Indeed, in Example 1 above $M$ and $M_{1}$ are isometrically isomorphic and, since $M$ contains the Dirichlet algebra $M_{1}$, the state spaces of $M$ and $M_{2}$ are equivalent to the state space of $C_{\mathbf{R}}(\Gamma)$.

We need to recall some concepts, full details of which may be found in Asimow and Ellis [1]. The centre of $A(K)$ consists of those functions $f \in A(K)$ such that for each $G \in A(K)$ there is some $h \in A(K)$ satisfying $h(x)=$ $f(x) g(x)$ for all $x \in \partial K$, where $\partial K$ denotes the set of extreme points of $K$. The sets of constancy in $\partial K$ for the central functions in $A(K)$ form 
the sets of extreme points of a family of faces $\left\{F_{\alpha}\right\}$ of $K$, called the Šilov decomposition for $A(K)$. The maximal subsets $E$ of $\partial K$ such that the centre of $A(\overline{c o} E)$ is trivial form the sets of extreme points of a family of faces $\left\{F_{\beta}\right\}$ of $K$ called the Bishop decomposition for $A(K)$. In the case when $K$ is the complex state space of a uniform algebra these decompositions are closely related to the corresponding classical decompositions.

If $Z$ is the complex state space of a function space $M$ then $\theta: M \rightarrow A(Z)$ will denote the real-linear homeomorphism defined by $\theta f(z)=\operatorname{re} z(f)$, noting that $\theta(u+i v)(\lambda x-i(1-\lambda) y)=\lambda u(x)+(1-\lambda) v(y)$ when $x, y \in X$ and $0 \leq \lambda \leq 1$. For this purpose we consider $X$ to be canonically embedded in $S$. $\theta_{1}, \theta_{2}$ will denote the corresponding maps for $M_{1}$ and $M_{2}$.

Theorem 1. Let $M$ be a complex function space on $X$ with complex state space $Z$, and let $M_{j}$ be uniform algebras with complex state spaces $Z_{j}, j=1,2$. If $M$ is isometrically isomorphic to $M_{1}$ and if $Z$ is equivalent to $Z_{2}$ then $M$ is a uniform algebra on $X$.

Proof. We first prove the result in the special case when $Z$ is real-equivalent to $Z_{2}$.

As in the discussion of the Examples above we may assume that $M_{1}, M_{2}$ are uniform algebras on $X$, and that

$$
M=\left\{l f: f \in M_{1}\right\}=\left\{u+i v \circ \psi: u+i v \in M_{2}\right\},
$$

where $l \in M$ with $|l|=1$ and $\psi: X \rightarrow X$ is a homeomorphism with $\psi^{2}$ equal to the identity map on the essential set for $M_{2}$. In order to prove that $M$ is an algebra it will be sufficient to show that $l \in M_{1}$, that is $l^{2} \in M$. Write $l=g+i h$ so that $g+i h=u+i v \circ \psi$ for some $u+i v \in M_{2}$. Since $M$ contains constants we must have $\bar{l} \in M_{1}$, so that $\bar{l}=l \bar{l}^{2} \in M$. Hence $g-i h=u_{1}+i v_{1} \circ \psi$ for some $u_{1}+i v_{1} \in M_{2}$. Consequently we obtain $g=u=u_{1}, h=v \circ \psi=-v_{1} \circ \psi$, so that $v=-v_{1}$ and $u-i v, u$ and $v$ belong to $M_{2}$. But then $l^{2}=g^{2}-h^{2}+2 i g h=2 u^{2}-1+2 i\left(\left(u \circ \psi^{-1}\right) v\right) \circ \psi$ belongs to $M$ because $u \circ \psi^{-1}$ belongs to $M_{2}$ (cf. [4]). Hence $M$ is an algebra.

We now turn to the general case where $Z$ and $Z_{2}$ are equivalent and $M=$ $\left\{l f: f \in M_{1}\right\}$, with $l \in M$. Firstly we identify the centres of $A(Z)$ and $A\left(Z_{1}\right)$. The centre of $A\left(Z_{1}\right)$ consists of the functions $\theta_{1}(u+i v)$ such that $u, v$ belong to $M_{1}$ and $u-v$ belongs to the essential ideal for $M_{1}$ (cf. [2, Theorem 1]).

Suppose that $\theta(u+i v)$ belongs to the centre of $A(Z)$. Then for each $a+i b \in$ $M$ we have $u a+i v b$ belongs to $M$, and since $1, i$ belong to $M$ we may deduce that $u$ and $v$ belong to $M$. If $a+i b \in M$ then we have $b-i a \in M$ and hence $u b-i v a$ and $v a+i u b$ belong to $M$. Consequently $u a+i v b+v a+i u b=$ $(u+v)(a+i b)$ belongs to $M$ and $u a+i v b-v a-i u b=(u-v)(a-i b)$ belongs to $M$. Conversely, reversing this argument, we see that $\theta(u+i v)$ belongs to the centre of $A(Z)$ whenever $(u+v) M$ and $(u-v) \bar{M}$ are contained in $M$. 
Therefore $\theta(u+i v)$ belongs to the centre of $A(Z)$ if and only if $f \in M_{1}$ implies that $(u+v) l f$ and $(u-v) \bar{l} \bar{f}$ belong to $M$, that is $(u+v) f$ and $(u-v) \bar{l}^{2} \bar{f}$ belong to $M_{1}$. Taking $f=1$ and also $f=\bar{l}^{2} \in M_{1}$, we see that $u+v, u-v, u$ and $v$ belong to $M_{1}$ whenever $\theta(u+i v)$ belongs to the centre of $A(Z)$. In this case taking $f=\bar{l}^{2} g$, where $g \in M_{1}$, we see that $(u-v) \bar{g} \in M_{1}$; since $(u-v) g$ belongs to $M_{1}$ it follows that $(u-v)$ re $g$ and $(u-v)$ im $g$ belong to $M_{1}$. The proof of [2, Theorem 1] now shows that $u-v$ belongs to the essential ideal $I_{1}$ of $M_{1}$, that is $\theta_{1}(u+i v)$ belongs to the centre of $A\left(Z_{1}\right)$. Conversely, if $u, v \in M_{1}$, and $u-v \in I_{1}$ then, for all $f \in M_{1}$, we have $(u+v) f,(u-v) \bar{l}^{2} \bar{f} \in M_{1}$, because $\bar{l}^{2} \bar{f} \in C_{\mathrm{C}}(X)$. Hence $\theta(u+i v)$ belongs to the centre of $A(Z)$, and we have shown that the centres of $A(Z)$ and $A\left(Z_{1}\right)$ may be identified.

We may assume without loss of generality that $X$ is the Silov boundary for both $M_{1}$ and $M$. Therefore we have shown that the Silov decompositions of $Y=X \cup-i X$ corresponding to $A(Z)$ and $A\left(Z_{1}\right)$ coincide. The Šilov decompositions of $Y$ for $A\left(Z_{1}\right)$, except for the singleton sets, consists of sets of the form $E_{\alpha} \cup-i E_{\alpha}$, where $E_{\alpha}$ belongs to the Šilov decomposition of $X$ for $M_{1}$ (cf. [3]). Now $M_{1} \mid E_{\alpha}$ and $M \mid E_{\alpha}$ are isometrically isomorphic, and we may apply the preceding reasoning to these spaces to conclude that the Bishop decompositions of $Y$ corresponding to $A(Z)$ and $A\left(Z_{1}\right)$ coincide.

The Bishop decomposition for $A(Z)$, except for singletons, consists of faces of the form $G_{\beta}=\operatorname{co}\left(F_{\beta} \cup-i F_{\beta}\right)$, where $F_{\beta} \cap X=E_{\beta}$ belongs to the Bishop decomposition for $M_{1}$. Moreover, if $g \in C_{\mathrm{C}}(X)$ is such that $g \mid E_{\beta}$ belongs to $M \mid E_{\beta}$ for all $\beta$, then $\bar{l} g \in C_{\mathrm{C}}(X)$ and $\bar{l} g\left|E_{\beta} \in M_{1}\right| E_{\beta}$ for all $\beta$ which implies that $\bar{l} g \in M_{1}$, and hence $g$ belongs to $M$. We can hence conclude that $M$ is an algebra if we can show that $l^{2} \mid E_{\beta}$ belongs to $M \mid E_{\beta}$ for all $\beta$.

Since $Z$ is equivalent to $Z_{2}$ the faces of the Bishop decompositions for $Z$ and $Z_{2}$ are equivalent. Therefore if we restrict attention to $M \mid E_{\beta}$ and $M_{1} \mid E_{\beta}$ we see that the complex state space $G_{\beta}$ of $M \mid E_{\beta}$ is equivalent to the complex state space of an antisymmetric uniform algebra $M_{3}$ (a restriction algebra of $M_{2}$ ). However in this case either $G_{\beta}$ is real-equivalent to $Z_{3}$ or is real-equivalent to the complex state space of $\bar{M}^{3}$. In either case the first part of the proof shows that $M \mid E_{\beta}$ is an algebra. Consequently $l^{2} \mid E_{\beta}$ belongs to $M \mid E_{\beta}$ and the proof of the theorem is complete.

We remark that the condition in Theorem 1 that $Z$ is equivalent to $Z_{2}$ is much weaker than the condition that $Z$ is real-equivalent to $Z_{2}$. In fact if $M$ is self-adjoint and if $Z$ is real-equivalent to $Z_{2}$ then, since $S$ is a split face of $Z, S_{2}$ must be a split face of $Z_{2}$ which implies that $M_{2}$ is a $C_{\mathrm{C}}(X)$ space. This conclusion need not hold when $Z$ and $Z_{2}$ are just equivalent, as the following example shows.

Example 3. Let $Z_{\Gamma}, Z_{\Gamma^{\prime}}$ denote respectively the complex state spaces of $P(\Gamma)$, $P\left(\Gamma^{\prime}\right)$, where $P\left(\Gamma^{\prime}\right)$ is the uniform algebra generated by the polynomials on 
$\Gamma^{\prime}=\{z \in \mathbf{C}:|z-3|=1\}$. Let $M=A_{\mathbf{C}}\left(Z_{\Gamma}\right)$ and $M_{2}=P\left(\Gamma \cup \Gamma^{\prime}\right)$. Then $Z_{2}$ is the convex hull of the disjoint closed split faces $Z_{\Gamma}$ and $Z_{\Gamma^{\prime}}$, while $Z$ is the convex hull of the disjoint closed split faces $Z_{\Gamma}$ and $-i Z_{\Gamma}$. Since $-i Z_{\Gamma}$ and $Z_{\Gamma^{\prime}}$ are equivalent so are $Z$ and $Z_{2}$. In this example $M$ is self-adjoint while the uniform algebra $M_{2}$ is not a $C_{\mathrm{C}}(X)$-space.

We note that it is easy to verify that no non-trivial uniform algebra can be isometrically isomorphic to a self-adjoint complex function space.

In the context of Theorem 1, Nagasawa's theorem [7] show that $M_{1}$ is unique in the sense that any two isometrically isomorphic uniform algebras are algebraically isomorphic. On the other hand $M_{2}$ need not be unique even if $M$ is a $C_{\mathrm{C}}(X)$-space (cf. [4, Example 2]). Our final result gives conditions under which $M_{2}$ is uniquely determined, up to complex conjugation. A related result appeared in Ellis and So [5, Corollary 6]).

Theorem 2. Let $M_{1}, M_{2}$ be uniform algebras with essential sets $X, Y$ respectively. If $Z_{1}$ and $Z_{2}$ are equivalent then $M_{2} \mid Y$ is isometrically isomorphic to $\left.\left(M_{1} \mid E\right) \otimes \bar{M}_{1} \mid X \backslash E\right)$, for some open and closed subset $E$ of $X$.

Proof. Let $\varphi: Z_{1} \rightarrow Z_{2}$ be an equivalence. The essential face for $Z_{1}$ has the form $\operatorname{co}(F \cup-i F)$, where $F$ is the closed convex hull of $X$ in $S_{1}$ (cf. [3, Proposition 17]). Since $\varphi$ maps the essential face of $Z_{1}$ onto the essential face of $Z_{2}$, and since $\operatorname{co}(F \cup-i F)$ is the complex state space of $M_{1} \mid X$, we can assume without loss of generality that $M_{1}$ and $M_{2}$ are essential uniform algebras, and that $X, Y$ are the Šilov boundaries of $M_{1}, M_{2}$ respectively.

If we write $E=\left\{x \in X: \varphi(x) \in S_{2}\right\}$ then $X=E \cup(X \backslash E)$ is a peak-set decomposition of $X$ for $M_{1}$ (cf. [5, Corollary 2]). Since $M_{1}$ is essential so are the algebras $M_{1} \mid E$ and $M_{1} \mid(X \backslash E)$ and hence $E$ (respectively $X \backslash E$ ) is the closure of the union of non-singleton maximal antisymmetric sets for $M_{1} \mid E$ (respectively $M_{1} \mid(X \backslash E)$ ) (cf. [6, page 65]).

The faces of the Bishop decomposition for $Z_{1}$ are the singletons $x,-i x$, where $x$ is a singleton member of the Bishop decomposition for $M_{1}$, together with faces of the form $\operatorname{co}\left(F_{\alpha} \cup-i F_{\alpha}\right)$, where $F_{\alpha}$ is the closed convex hull in $S_{1}$ of a non-singleton member of the Bishop decomposition for $M_{1}$. Now each $\operatorname{co}\left(F_{\alpha} \cup-i F_{\alpha}\right)$ is mapped by $\varphi$ onto a corresponding member $\operatorname{co}\left(G_{\alpha} \cup-i G_{\alpha}\right)$ of the Bishop decomposition for $Z_{2}$. Consequently $\operatorname{co}(E \cup-i E)$ is mapped onto a face of the form $\operatorname{co}(H \cup-i H)$, and similarly for $\overline{\mathrm{co}}((X \backslash E) \cup-i(X \backslash E))$. Since the Bishop decompositions determine $M_{1}$ and $M_{2}$, and since we have $M_{1} \mid\left(F_{\alpha} \cap X\right)=\left\{f \circ \varphi: f \in M_{2} \mid\left(G_{\alpha} \cap Y\right)\right\}$ whenever $F_{\alpha} \cap E$ is non-empty we see that $M_{1} \mid E=\left\{f \circ \varphi: f \in M_{2} \mid \varphi(E)\right\}$. Therefore $M_{1} \mid E$ is isometrically isomorphic to $M_{2} \mid \varphi(E)$. Similarly we may prove that $\bar{M}_{1} \mid(X \backslash E)$ is isometrically isomorphic to $M_{2} \mid(Y \backslash \varphi(E))$. 


\section{REFERENCES}

1. L. Asimow and A. J. Ellis, Convexity theory and its applications in functional analysis, London Math. Soc. Monograph 16, Academic Press, London, 1980.

2. A. J. Ellis, On facially continuous functions in function algebras, J. London Math. Soc. (2) 5(1972), 561-564.

3. Central decompositions and the essential set for the space $A(K)$, Proc. London Math. Soc. (3) 26(1973), 564-576.

4. __ Equivalence for complex state spaces of function spaces, Bull. London Math. Soc. 19(1987), 359-362.

5. A. J. Ellis and W. S. So, Isometries and the complex state spaces of uniform algebras, Math. Z. 195(1987), 119-125.

6. G. M. Leibowitz, Lectures on complex function algebras, Scott-Foresman, Glenview, 1969.

7. M. Nagasawa, Isomorphisms between commutative Banach algebras with an application to rings of analytic functions, Kōdai Math. Sem. Rep. 11(1959), 182-188.

Department of Mathematics, University of Hong Kong, Pokfulam Road, Hong Kong 